\title{
UNIVERSITYOF BIRMINGHAM

\section{Functional consequences of germline mutations in a novel non-RET medullary thyroid cancer susceptibility gene}

Read, Martin; Smith, Joel; Smith, Vicki; Bosseboeuf, Emy; Wake, Naomi; Watkinson, John; Wallis, Yvonne; Maher, Eamonn; McCabe, Christopher; Woodward, Emma

DOI:

10.1530/endoabs.38.0C5.1

\section{Document Version}

Publisher's PDF, also known as Version of record

Citation for published version (Harvard):

Read, M, Smith, J, Smith, V, Bosseboeuf, E, Wake, N, Watkinson, J, Wallis, Y, Maher, E, McCabe, C \&

Woodward, E 2015, 'Functional consequences of germline mutations in a novel non-RET medullary thyroid cancer susceptibility gene', Endocrine Abstracts, vol. 38, OC5.1. https://doi.org/10.1530/endoabs.38.OC5.1

Link to publication on Research at Birmingham portal

\section{General rights}

Unless a licence is specified above, all rights (including copyright and moral rights) in this document are retained by the authors and/or the copyright holders. The express permission of the copyright holder must be obtained for any use of this material other than for purposes permitted by law.

- Users may freely distribute the URL that is used to identify this publication.

- Users may download and/or print one copy of the publication from the University of Birmingham research portal for the purpose of private study or non-commercial research.

- User may use extracts from the document in line with the concept of 'fair dealing' under the Copyright, Designs and Patents Act 1988 (?)

- Users may not further distribute the material nor use it for the purposes of commercial gain.

Where a licence is displayed above, please note the terms and conditions of the licence govern your use of this document.

When citing, please reference the published version.

Take down policy

While the University of Birmingham exercises care and attention in making items available there are rare occasions when an item has been uploaded in error or has been deemed to be commercially or otherwise sensitive.

If you believe that this is the case for this document, please contact UBIRA@lists.bham.ac.uk providing details and we will remove access to the work immediately and investigate. 


\section{$0 \mathrm{C} 4.4$}

Hyperinsulinaemia due to inhibition of $5 \alpha$-reductases is ameliorated by liver-selective glucocorticoid receptor antagonism in diet-induced obesity

Tracy C S Mak, Dawn E W Livingstone, Brian R Walker \& Ruth Andrew University of Edinburgh, Edinburgh, UK.

Background

$5 \alpha$-reductase $1(5 \alpha \mathrm{R} 1)$ metabolises steroids such as glucocorticoids and androgens, and is highly expressed in murine liver. Genetic disruption of $5 \alpha \mathrm{R} 1$ leads to adverse metabolic changes in mice. We hypothesised that dutasteride, a $5 \alpha \mathrm{R}$ inhibitor, induces insulin resistance in mice, as in humans, and this effect is underpinned by increased hepatic glucocorticoid action; an experimental paradigm was set up using A-348441, a liver-selective glucocorticoid receptor (GR) antagonist, and then utilised to assessed the contribution of increased hepatic glucocorticoid action to the metabolic consequences of dutasteride. Methods

C57BL6/J male mice ( $n=8-15 /$ group; age 12 weeks) were given high fat (HF), HF with A-348441 (KaroBio), HF + dutasteride (Dut), or HF + Dut + A-348441 diet for 4 weeks. Glucose tolerance tests (GTT) were performed at week 3, with mice culled at week 4. Plasma insulin and corticosterone were measured by ELISA and plasma glucose spectrophotometrically. Data are mean \pm s.E.M., ${ }^{\#} P<0.05$ vs HF diet and ${ }^{\$} P<0.05$ vs HF + Dut diet.

Results

Plasma corticosterone concentrations were not changed by A-348441, supporting liver-selective GR antagonism. A-348441 improved metabolic health of mice receiving a HF diet, preventing HF-induced bodyweight gain $(34.3 \pm 0.5 \mathrm{~g}$ vs $\left.31 \pm 0.8 \mathrm{~g}^{\#}\right)$, and total white adipose depot weight gain $(2.46 \pm 0.1 \mathrm{~g}$ vs $1.58 \pm$ $\left.0.1 \mathrm{~g}^{\#}\right)$, and attenuating HF-induced elevations in fasting plasma insulin, fasting glucose and insulin response to GTT (lowered by $52^{\#}, 25^{\#}$, and $44 \%^{\#}$ respectively) . Inhibition of $5 \alpha$ Rs with dutasteride impaired insulin sensitivity, with increased insulin response to GTT but did not change body weight, total adipose depot weight, fasting insulin, fasting glucose, or glucose response to GTT; A-348441 reduced this hyperinsulinaemia $\left(235.9 \pm 17 \mathrm{ng} / \mathrm{ml}\right.$ per $\min$ vs $329.3 \pm 16^{\#} \mathrm{ng} / \mathrm{ml}$ per min vs $198.4 \pm 25^{\$} \mathrm{ng} / \mathrm{ml}$ per min).

Conclusions

Liver-specific GR antagonism ameliorates the metabolic consequences of acute diet-induced obesity. Hyperinsulinaemia caused by inhibition of $5 \alpha$ Rs was ameliorated by A-348441, suggesting that hepatic glucocorticoid action plays a substantial role in metabolic dysfunction caused by $5 \alpha \mathrm{R}$ inhibition. Moreover, targeting hepatic GR may be beneficial in maintaining metabolic homeostasis in diet-induced obesity.

DOI: 10.1530/endoabs.38.OC4.4

0C4.5

Glucagon increases energy expenditure independently of brown adipose tissue activation in humans

Chioma Izzi-Engbeaya ${ }^{1}$, Victoria Salem ${ }^{1}$, Rajveer Atkar ${ }^{1}$,

Christopher Coello ${ }^{2}$, David Thomas ${ }^{3}$, Alexander Comninos ${ }^{1}$,

Adam Buckley ${ }^{1}$, Eugenii Rabiner ${ }^{2}$, Roger Gunn ${ }^{2}$, Tricia Tan ${ }^{1}$,

Stephen Bloom ${ }^{1} \&$ Waljit Dhillo ${ }^{1}$

${ }^{1}$ Section of Investigative Medicine, Imperial College London, London, UK;

${ }^{2}$ Imanova Imaging Centre, London, UK; ${ }^{3}$ Department of Computing,

Imperial College London, London, UK.

Background

Obesity is a global health concern. Elevating energy expenditure (EE) would be a highly effective treatment approach to treat obesity but no current drugs can safely achieve this. Cold exposure potently increases EE through brown adipose tissue (BAT) thermogenesis in humans. Glucagon elevates EE via BAT in rodents but the mechanism in humans is unknown. We investigated for the first time the mechanism by which glucagon increases EE in humans.

Methods

Eleven volunteers underwent measurement of EE using an indirect calorimeter at the start and end of three interventions: i) cold exposure; ii) control (vehicle) infusion at $23{ }^{\circ} \mathrm{C}$; and iii) glucagon infusion at $23{ }^{\circ} \mathrm{C}$. On each visit thermal images of the neck were taken - an increase in temperature is a non-invasive measure of increased BAT activity. All 11 volunteers also underwent a FDG PET-CT scan with cold exposure. In those in which this confirmed cold-induced BAT activity $(n=8)$, they had a second PET-CT scan with either vehicle $(n=4)$ or glucagon $(n=4)$ infusion $\left(23^{\circ} \mathrm{C}\right)$

Results

EE rose by $14 \%$ with cold exposure and $15 \%$ following glucagon infusion $(P<0.05$ vs control). BAT depots identified on the cold scan had significantly
$(4 \times)$ higher metabolic activity than on the vehicle or glucagon infusion scans, which were not significantly different from each other. There was a $0.31{ }^{\circ} \mathrm{C}$ rise $(P<0.001)$ in neck temperature on thermal images after cold exposure in the BAT positive cohort but not after glucagon or vehicle infusion.

Conclusions

Glucagon and cold exposure have a similar effect in stimulating energy expenditure but glucagon has no effect on the metabolic activity of classical adult supraclavicular BAT compared with cold exposure. This information is of importance to the development of better targeted and safe treatments designed to combat obesity through upregulation of energy expenditure.

DOI: 10.1530/endoabs.38.OC4.5

OC4.6

Cardiac fibrosis and the balance between glucocorticoid and mineralocorticoid receptors signalling

Rachel Richardson ${ }^{1,2}$, Emma Batchen ${ }^{1}$, Rowan Darroch ${ }^{1}$,

Kathleen Scullion ${ }^{1}$, Ewa Rog-Zielinska ${ }^{1,3}$, Christopher Kenyon ${ }^{1}$, Gillian Gray ${ }^{1}$ \& Karen Chapman ${ }^{1}$

${ }^{1}$ University of Edinburgh, Edinburgh, UK; ${ }^{2}$ Newcastle University,

Newcastle, UK; ${ }^{3}$ Imperial College London, London, UK.

Specific variations in the human glucocorticoid receptor (GR) gene associate with increased cardiovascular disease risk. GR signalling is essential for cardiac maturation in utero and adult mice with cardiomyocyte and vascular smooth muscle deletion of GR (SMGRKO mice) have cardiac hypertrophy, fibrosis and impaired function. Intriguingly, levels of left ventricle (LV) mRNA encoding the mineralocorticoid receptor (MR), which is pro-fibrotic in heart, rise postnatally in SMGRKO mice in parallel with the development of cardiac fibrosis. Here, the benefit of MR antagonism in limiting cardiac fibrosis was assessed in SMGRKO mice.

SMGRKO mice (generated via SM22 $\alpha$-Cre mediated deletion of GR) and control (Cre - ) littermates were treated from birth with vehicle or $20 \mathrm{mg} / \mathrm{kg}$ per day spironolactone, an MR antagonist, administered in the drinking water to lactating dams until weaning then to offspring ( $n=10-13$ /group). At 8 weeks of age, hearts were collected for histology and mRNA profiling. Data were analysed by twoway ANOVA with Tukey's multiple comparisons test.

Heart weight in male SMGRKO mice was higher than controls irrespective of spironolactone treatment $(P<0.01)$. Interestingly, spironolactone modestly reduced heart weight in both genotypes $(P<0.05)$.

PicroSirius Red staining showed greater collagen levels in LV of SMGRKO mice $(P<0.001)$; spironolactone treatment reduced the magnitude of this genotypic difference. Although spironolactone did not prevent the increase in LV levels of mRNA encoding MR or profibrotic factors (connective tissue growth factor, collagen $1 \alpha 2$ and collagen $3 \alpha 1)$ in SMGRKO mice, it did attenuate collagen $1 \alpha 2$ mRNA increases $(P<0.05)$

In conclusion, the modulatory effects of spironolactone on pro-fibrotic signalling suggest that elevated MR contributes to the pro-fibrotic cardiac phenotype discovered in SMGRKO mice. Consequently, MR antagonism may benefit individuals with particular variants of the GR gene. Spironolactone effects on heart weight indicate a role for MR in early life cardiac growth and SMGRKO mice are, potentially, a useful new model to investigate MR-dependent cardiac fibrosis.

DOI: $10.1530 /$ endoabs.38.OC4.6

\section{Thyroid and parathyroid \\ 0C5.1}

Functional consequences of germline mutations in a novel non-RET medullary thyroid cancer susceptibility gene

Martin Read ${ }^{1}$, Joel Smith ${ }^{1}$, Vicki Smith ${ }^{1}$, Emy Bosseboeuf ${ }^{1}$, Naomi Wake ${ }^{1}$, John Watkinson ${ }^{1,2}$, Yvonne Wallis ${ }^{1,3}$, Eamonn Maher ${ }^{1,4}$ Christopher McCabe ${ }^{1}$ \& Emma Woodward ${ }^{1,3}$

${ }^{1}$ Centre for Endocrinology, Diabetes and Metabolism, Institute of Metabolism and Systems Research, University of Birmingham, Birmingham, UK; ${ }^{2}$ University Hospitals Birmingham NHS Foundation Trust, Birmingham, UK; ${ }^{3}$ Birmingham Women's NHS Foundation Trust, Birmingham, UK; ${ }^{4}$ Department of Medical Genetics, University of Cambridge, Cambridge, UK.

Whilst the majority of familial medullary thyroid cancer (MTC) is caused by germline mutations of the RET proto-oncogene, there are families and individuals 
with predisposition to MTC in whom no RET mutation has been identified (nonRET MTC). Recently, we identified novel mutations in a single gene termed MTC2 in non-RET MTC individuals by whole exome sequencing. The precise role of these MTC2 germline mutations in MTC tumorigenesis is however unclear. Here, we examined the functional consequences of MTC2 mutants V128L and G318Afs*22 to determine their roles in MTC. Luciferase (LUC) reporter assays showed that MTC2-V128L retained transcriptional activity with a significant increase in LUC activity in response to steroid hormone receptoragonist DPN in HCT116 (3.7-fold; $P<0.01$ ) and MCF-7 (1.8-fold; $P<0.05$ ) cells. In contrast, MTC2-G318Afs*22 was incapable of inducing LUC activity in either cell line $(P=\mathrm{NS})$. Furthermore, MTC2-G318Afs $* 22$ failed to inhibit ER $\alpha$ driven luciferase activity in response to either $17 \beta$-estradiol $\left(E_{2}\right)$ or $E R \alpha$-agonist $\mathrm{PPT}$, or restrain ER $\alpha$-driven proliferation of MCF7 cells ( $P=\mathrm{NS}$ compared to $\mathrm{ER} \alpha$ alone). In contrast, WT MTC2 and MTC2-V128L inhibited ER $\alpha$-driven LUC activity $(>60 \% ; P<0.01)$ and cell proliferation $(>30 \% ; P<0.05)$. As RET expression is known to be stimulated by oestrogen, we then determined the influence of MTC2 mutants on RET in $\mathrm{E}_{2^{-}}$and PPT-treated HCT116 cells. In contrast to WT MTC2, MTC2-G318Afs*22 was unable to oppose ER $\alpha$ stimulation of the RET proto-oncogene at both the mRNA and protein level $(P=\mathrm{NS}$ compared to $\mathrm{ER} \alpha$ alone). Treatment with anti-oestrogen 4-hydroxytamoxifen was however capable of inhibiting $\mathrm{E}_{2}$-induced RET mRNA expression in cells with MTC2-G318Afs*22. Together these data indicate an emerging role for MTC2 as a novel susceptibility gene in non-RET MTC development, especially as MTC2 mutant G318Afs*22 was associated with higher RET levels. These results also suggest that anti-oestrogens might represent a promising therapeutic strategy for MTC individuals with defective MTC2. DOI: 10.1530 /endoabs.38.OC5.1

\section{C5.2}

A novel, missense, mutation (P81R) in the TRH receptor gene in congenital central hypothyroidism

Olympia Koulouri ${ }^{1}$, Adeline Nicholas ${ }^{1}$, Erik Schoenmakers ${ }^{1}$, Jacek Mokrosinski ${ }^{1}$, Frances Lane ${ }^{2}$, Trevor Cole ${ }^{2}$, Jeremy Kirk ${ }^{3}$, Sadaf Farooqi ${ }^{1}$, Krishna Chatterjee ${ }^{1}$, Mark Gurnell ${ }^{1}$ \& Nadia Schoenmakers ${ }^{1}$

${ }^{1}$ Metabolic Research Laboratories, Wellcome Trust-MRC Institute of Metabolic Science, Addenbrooke's Hospital, University of Cambridge, Cambridge, UK; ${ }^{2}$ West Midlands Regional Genetics Service, Birmingham Womens Hospital NHS Foundation Trust, Birmingham, UK; ${ }^{3}$ Department of Endocrinology, Birmingham Children's Hospital, Birmingham, UK.

Background

Congenital, isolated, central, hypothyroidism $(\mathrm{CCH})$, is rare and evades diagnosis on TSH-based congenital hypothyroidism screening programmes in the UK. Genetic ascertainment is therefore paramount in enabling prompt diagnosis and treatment of familial cases. Recognised causes include TSHB and IGSFI gene defects, with only two previous reports of biallelic, highly disruptive (nonsense R17X, in-frame deletion and missense; p.S115-T117del+T118), mutations in the $T R H R$ gene. Here, we describe the first homozygous missense mutation in TRHR, associated with a typical phenotype

Case

A female infant from a consanguineous Pakistani family, presented with prolonged neonatal jaundice and was found to have central hypothyroidism (TSH $2.2 \mathrm{mU} / \mathrm{l}$, NR $0.4-3.5$ and free $\mathrm{T}_{4} 7.9 \mathrm{pmol} / \mathrm{l}$, NR 10.7-21.8), with otherwise normal pituitary function. With TSHB or IGSF1 mutations being usually associated with profound or X-linked $\mathrm{CCH}$, a TRHR mutation was sought. Results

Sequencing identified a homozygous mutation (P81R) in TRHR, substituting arginine for a proline residue in transmembrane helix 2 (TM2) which is highly conserved amongst G-protein coupled receptors (GPCRs). Functional studies showed that although the mutant receptor was expressed and localised to the cell membrane normally, its ability to bind radiolabelled TRH and signal via Gq $\alpha$ was markedly impaired, likely due to disruption of structure of TM2.

Conclusion

We describe the first deleterious, missense TRHR defect associated with moderate $\mathrm{CCH}$. Importantly, the location of the mutated amino acid (proline 81) highlights a previously unanticipated functional importance of the TM2 in mediating hormone binding and receptor activation. Future identification of other, naturallyoccurring, TRHR mutations may map the molecular basis of ligand binding and activation of TRHR which are poorly understood.

DOI: 10.1530 /endoabs.38.OC5.2

\section{$0 \mathrm{C} 5.3$}

Use of ${ }^{11} \mathrm{C}$-methionine PET to localise parathyroid

adenoma/hyperplasia: a single centre experience

Ben Challis ${ }^{2}$, Ziauddin Saad ${ }^{1}$, H K Cheow ${ }^{1}$, John Buscombe ${ }^{1}$ \&

Helen Simpson ${ }^{1}$

${ }^{1}$ Cambridge University Hospitals NHS Foundation Trust, Cambridge, UK;

${ }^{2}$ University of Cambridge, Cambridge, UK.

\section{Introduction}

It is established practice to localise parathyroid lesions preoperatively using ultrasound (US) and sestaMIBI (MIBI). Whilst these imaging techniques have good sensitivity/specify, there are patients in which imaging does not localise a parathyroid lesion. ${ }^{11} \mathrm{C}$-Methionine PET (MET PET) is an imaging modality where ${ }^{11} \mathrm{C}$-methionine, a radioactive tracer, is taken up at sites of protein/peptide synthesis and has been demonstrated to be effective in localising parathyroid lesions. We therefore investigated the clinical utility of this imaging technique at our centre.

Methods

All patients had biochemistry prior to imaging thought to be consistent with primary hyperparathyroidism. Criteria to undergo PET imaging were inability of conventional imaging to identify a parathyroid lesion, potential intrathyroidal parathyroid lesion, and three patients where mediastinal disease was suspected. Twenty patients underwent MET PET over an 18-month period.

Results

MET PET identified a parathyroid lesion in 14/20 patients. Three out of three of these were demonstrated to be mediastinal lesions, leading to a parathyroid adenoma being successfully resected by sternotomy. 11/20 demonstrated disease in the neck. Of these 3/11 parathyroid lesions were very deep in the neck adjacent to vertebrae/oesophagus and not seen with US/sestaMIBI. In 2/11 patients MET PET demonstrated intrathyroidal parathyroid lesions and patients underwent hemithyroidectomy. All parathyroid lesions were confirmed on histology (13 adenoma and one hyperplasia). Of the $6 / 20$ who had negative imaging, one now has a diagnosis of sarcoidosis with elevated 1,25-dihydroxycholecalciferol, one underwent bilateral neck exploration and histology demonstrated parathyroid hyperplasia. The remaining four patients are still being investigated with working diagnoses of $\mathrm{FHH}$ in three patients.

Discussion

MET PET is a useful additional functional imaging technique when conventional imaging fails to localise a lesion, where mediastinal disease is suspected or intrathyroidal disease needs confirmation. This can particularly helpful when deciding to refer patients for major surgery.

DOI: $10.1530 /$ endoabs.38.OC5.3

\section{$0 \mathrm{C5.4}$}

A novel modulator of cellular invasion and metastasis in endocrine cancer

Rachel Watkins ${ }^{1}$, Waraporn Imruetaicharoenchoke ${ }^{1}$, Neil Sharma ${ }^{1}$, Erica Gentillin ${ }^{2}$, Emy Bosseboeuf ${ }^{1}$, Perkin Kwan ${ }^{1}$, Rachel Fletcher ${ }^{1}$, Hisham Mehanna ${ }^{1}$, Kristien Boelaert ${ }^{1}$, Martin Read ${ }^{1}$, Vicki Smith ${ }^{1}$ \& Christopher McCabe

${ }^{1}$ University of Birmingham, Birmingham, UK; ${ }^{2}$ University of Ferrara, Ferrara, Italy.

Metastasis is a multistep process responsible for the majority of endocrine cancer deaths. Central to the ability of cells to move is the recruitment of actin fibres at the periphery of the cell by key proteins, especially the cortical actin binding protein cortactin. A full understanding of cortactin function is required in order to address metastatic cell activity within endocrine cancer. We used IP-MS to discover protein binding partners, and now identify the proto-oncogene PBF as a new functional binding partner of cortactin, whose expression has recently been correlated with thyroid and breast cancer metastasis, and with colon cancer extramural vascular invasion. We show that cortactin and PBF interact and co-localise through immunofluorescence and Proximity Ligation Assays, and that this occurs within or close to the plasma membrane, and preferentially at the leading edge of migrating cells. Oncogenic expression of PBF induced potent cell invasion and migration in thyroid TPC-1 $(P=0.01)$, breast MCF-7 $(P<0.001)$ and colorectal HCT116 cells $(P<0.001)$, which was entirely abrogated by the knockdown of cortactin expression. In $n=43$ matched papillary thyroid cancers, cortactin was significantly upregulated at the mRNA $(P=0.022)$ and protein $(P=0.045)$ levels, particularly in more aggressive tumours, and significantly correlated with $\mathrm{PBF}$ expression. We also demonstrate the interaction between PBF and cortactin through co-immunoprecipitation assays and reveal that artificially targeting PBF to the plasma membrane results in increased cortactin binding, entirely blocking endogenous cellular invasion. Thus, we identify a new modulator of cortactin 